\title{
Ipofisite da inibitori dei checkpoint immunologici
}

\author{
Alice Nervo ${ }^{1}$. Valentina D'Angelo ${ }^{1} \cdot$ Emanuela Arvat $^{1}$
}

Accettato: 30 settembre 2020 / Pubblicato online: 13 maggio 2021

(c) The Author(s) 2021

\section{Introduzione}

L'ipofisite correlata al trattamento con inibitori dei checkpoint immunologici (ICI-I) rappresenta un possibile evento avverso (AE) legato all'impiego di questi farmaci, recentemente introdotti in campo oncologico con risultati sorprendenti. L'incidenza di tale complicanza endocrina varia a seconda delle casistiche considerate, sia per l'impiego di criteri diagnostici non omogenei tra loro, sia per la consapevolezza crescente riguardo la sua possibile insorgenza che ha contribuito all'incremento diagnostico. L'ICI-I si riscontra maggiormente in corso di terapia con anticorpi anti-CTLA4 (3,2\% con ipilimumab, secondo una recente metanalisi) mentre è più rara durante anti-PD1 $(0,4 \%$ con pembrolizumab o nivolumab) o anti-PD-L1 $(<0,1 \%$ con avelumab, durvalumab o atezolizumab). È frequentemente riscontrata anche in corso di terapia di combinazione anti-CTLA4/anti-PD1 (6,4\%) [1].

Insorge dopo un tempo mediano di $2-3$ mesi (range 4 settimane - 19 mesi) durante anti-CTLA4, in epoca più tardiva in corso di anti-PD1 o PD-L1 (3-6 mesi) e più precocemente durante terapia di combinazione (30 giorni). La sua comparsa sembra essere dose-dipendente e l'età avanzata costituisce un fattore di rischio aggiuntivo. Diversamente dalla forma autoimmune classica, colpisce maggiormente il sesso maschile (4:1).

Come per altri ICI-AEs, alcuni dati suggeriscono una maggiore efficacia antineoplastica del trattamento oncologico nei pazienti che sviluppano ICI-I: in un gruppo di pazienti con melanoma metastatico trattati con ipilimumab, è stata

osservata una migliore OS in coloro che avevano manifestato questa complicanza, sebbene il dato debba essere ancora confermato su casistiche più ampie [2].

\section{Patogenesi}

Il meccanismo patogenetico sottostante non è ancora completamente noto. Dopo somministrazione di anti-CTLA4, nei modelli murini è stata osservata un'infiltrazione linfocitaria a carico della ghiandola ipofisaria. Si è ipotizzato che l'interazione dei farmaci anti-CTLA4 con CTLA4, fisiologicamente espresso a livello ipofisario anche nell'uomo, scatenerebbe un processo infiammatorio attraverso reazioni immunitarie di tipo II e IV. Sono meno chiari i processi patogenetici legati all'insorgenza di ICI-I in corso di antiPD1 o anti-PD-L1. Inoltre, non è ancora del tutto definito il ruolo degli anticorpi anti-ipofisi, la cui comparsa è stata evidenziata nei pazienti con ICI-I [2,3].

\section{Presentazione clinica}

Il quadro clinico all'esordio è piuttosto aspecifico; nel paziente oncologico avanzato, la diagnosi è resa ancora più difficoltosa dalla compresenza di sintomi sistemici correlati alla patologia tumorale. La presenza di ICI-I andrebbe sospettata in caso di comparsa o peggioramento di astenia e/o cefalea; è possibile l'insorgenza di disturbi visivi conseguenti a compressione del chiasma ottico $\mathrm{o}$, ancor più raramente, di poliuria/polidipsia [4].

\section{Alterazioni biochimiche/ormonali}

I più comuni deficit ormonali riscontrati sono l'ipotiroidismo ( $\sim 85 \%)$, l'iposurrenalismo ( $\sim 80 \%)$ e l'ipogonadismo 


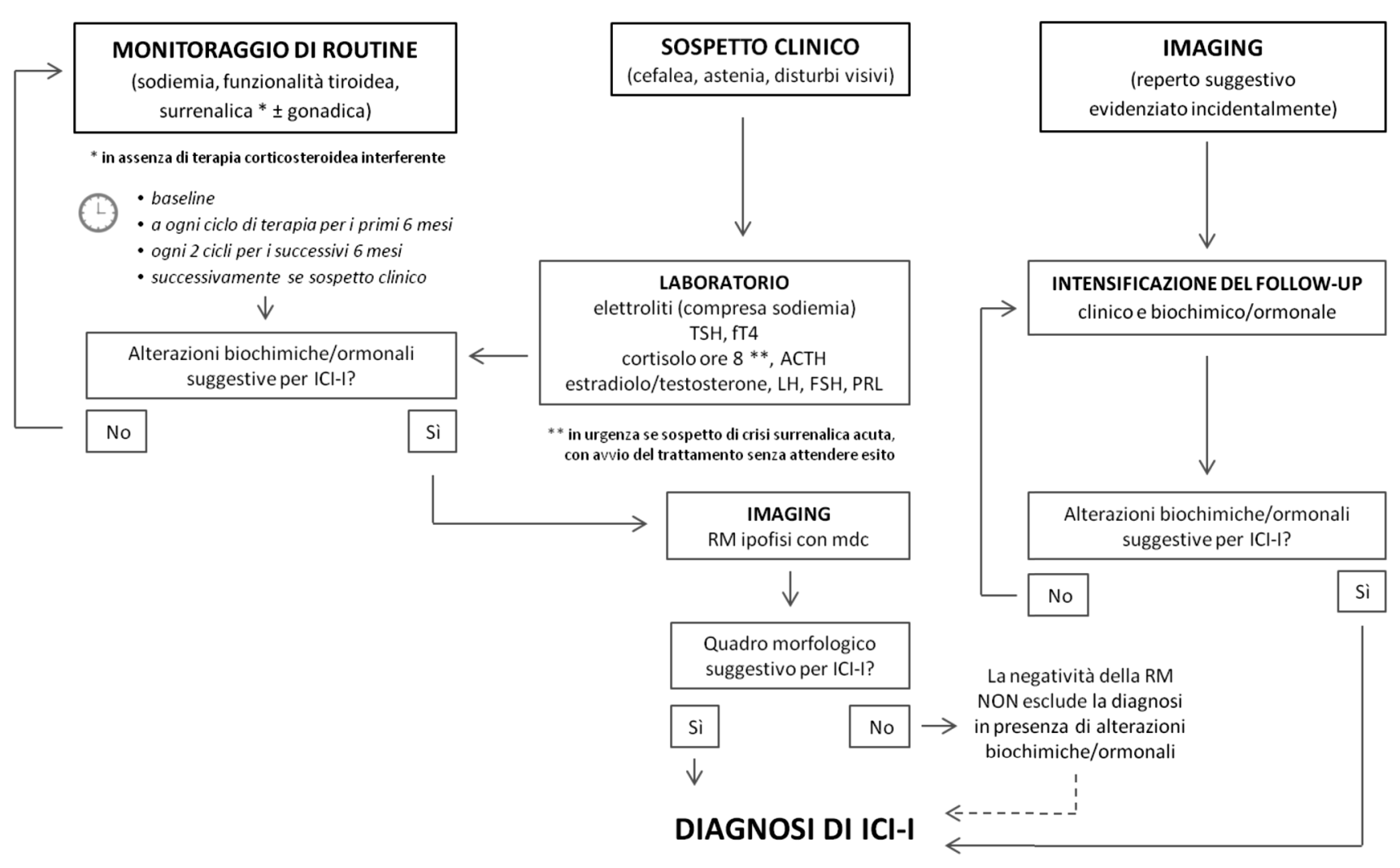

\section{GESTIONE}

CRISI SURRENALICA ACUTA: idrocortisone ev -> terapia per os a scalare fino a dosaggio sostitutivo

IPOSURRENALISMO SECONDARIO: terapia sostitutiva immediata IPOTIROIDISMO SECONDARIO: possibile rivalutazione nel tempo IPOGONADISMO SECONDARIO: possibile rivalutazione nel tempo

\section{FOLLOW-UP}

Compenso funzionale delle alterazioni note Ricerca di eventuali altri deficit insorti

CEFALEA REFRATTARIA/DISTURBI DEL CAMPO VISIVO: terapia steroidea ad alto dosaggio

Fig. 1 Iter diagnostico-terapeutico suggerito in caso di ICI-I

( $\sim 75 \%)$ secondari; i valori di prolattina risultano più frequentemente ridotti, mentre i dati sul deficit di GH non sono univoci. È comune il rilievo di iponatremia (fino al 50\%), raro il diabete insipido. La condizione di iposurrenalismo, potenzialmente pericolosa per la vita, è generalmente definitiva, mentre la persistenza di deficit a carico dell' asse tiroideo o gonadico è minore ( $45 \mathrm{e} 35 \%$ dei casi, rispettivamente) [2].

\section{Diagnostica per immagini}

Il quadro radiologico associato a ICI-I, transitorio in oltre i due terzi dei casi, può precedere la comparsa di alterazioni biochimiche ed è generalmente meno compromesso rispetto alla forma autoimmune classica. Alla risonanza magnetica (RM) è possibile osservare un incremento dimensio- nale diffuso della ghiandola e, talvolta, del peduncolo ipofisario; la distribuzione del mezzo di contrasto può essere uniforme o disomogenea. La negatività della RM non esclude la diagnosi di ICI-I. L'imaging morfologico permette, inoltre, di identificare l'eventuale presenza di metastasi ipofisarie, anch'esse potenzialmente responsabili di un quadro di ipopituitarismo [2, 3].

\section{Gestione diagnostico-terapeutica}

L'iter diagnostico-terapeutico proposto in caso di ICI-I è sintetizzato nella Figura 1.

Si consiglia di effettuare un monitoraggio clinico e biochimico/ormonale sistematico, specialmente durante antiCTLA4, all'inizio e in corso di terapia. In caso di riscontro radiologico incidentale di anomalie ipofisarie, è opportuno intensificare ulteriormente i controlli. 
I criteri diagnostici di ICI-I non sono attualmente standardizzati: secondo alcune proposte, la diagnosi di ICI-I può essere effettuata in caso di $\geq 2$ deficit ipofisari (comprendenti ipotiroidismo o iposurrenalismo secondario) oppure $\geq 1 \mathrm{de}$ ficit ipofisario associato ad alterazioni RM in presenza di sintomi suggestivi [3].

L'insorgenza di ICI-I non controindica la prosecuzione dell'immunoterapia, che può essere eventualmente posticipata in base alle condizioni cliniche del paziente.

In presenza di iposurrenalismo, specie in corso di crisi surrenalica acuta, la terapia cortisonica deve essere avviata in modo tempestivo. È inoltre fondamentale educare i pazienti alla gestione della terapia sostitutiva in caso di stress psico-fisico o impossibilità all'assunzione della terapia orale.

L'avvio della terapia sostitutiva tiroidea e gonadica è meno urgente e, se il deficit è di lieve entità, può eventualmente essere posticipato proseguendo uno stretto follow-up, dal momento che è possibile la ripresa di questi due assi anche nel giro di pochi mesi [4].

La terapia steroidea ad alto dosaggio è indicata in caso di cefalea refrattaria o disturbi del campo visivo [2].

\section{Conclusioni}

Specie in corso di anti-CTLA4, l'ICI-I deve essere ricercata in modo sistematico allo scopo di identificare e gestire precocemente le alterazioni cliniche e biochimiche correlate, permettendo la prosecuzione dell'onco-immunoterapia stessa. È pertanto fondamentale la gestione multidisciplinare di questi pazienti, basata su una stretta collaborazione tra oncologo ed endocrinologo.

Funding Note Open access funding provided by Università degli Studi di Torino within the CRUI-CARE Agreement.
Conflitto di interesse Le autrici Alice Nervo, Valentina D'Angelo e Emanuela Arvat dichiarano di non avere conflitti di interesse.

Consenso informato Lo studio presentato in questo articolo non ha richiesto sperimentazione umana.

Studi sugli animali Le autrici di questo articolo non hanno eseguito studi sugli animali.

Nota della casa editrice Springer Nature rimane neutrale in riguardo alle rivendicazioni giurisdizionali nelle mappe pubblicate e nelle affiliazioni istituzionali.

Open Access This article is licensed under a Creative Commons Attribution 4.0 International License, which permits use, sharing, adaptation, distribution and reproduction in any medium or format, as long as you give appropriate credit to the original author(s) and the source, provide a link to the Creative Commons licence, and indicate if changes were made. The images or other third party material in this article are included in the article's Creative Commons licence, unless indicated otherwise in a credit line to the material. If material is not included in the article's Creative Commons licence and your intended use is not permitted by statutory regulation or exceeds the permitted use, you will need to obtain permission directly from the copyright holder. To view a copy of this licence, visit http://creativecommons.org/licenses/by/4.0/.

\section{Bibliografia}

1. Barroso-Sousa R, Barry WT, Garrido-Castro AC et al (2018) Incidence of endocrine dysfunction following the use of different immune checkpoint inhibitor regimens: a systematic review and meta-analysis. JAMA Oncol 4(2):173-182

2. Albarel F, Castinetti F, Brue T (2019) Management of endocrine disease: immune check point inhibitors-induced hypophysitis. Eur J Endocrinol 181(3):R107-R118

3. Agrawal L, Bacal A, Jain S et al (2020) Immune checkpoint inhibitors and endocrine side effects, a narrative review. Postgrad Med 132(2):206-214

4. Castinetti F, Albarel F, Archambeaud F et al (2019) French Endocrine Society Guidance on endocrine side effects of immunotherapy. Endocr-Relat Cancer 26(2):G1-G18 